Portland State University

PDXScholar

1990

\title{
Intelligibility of spondees via bone conduction at elevated presentation levels
}

Pamela S. Ritchie

Portland State University

Follow this and additional works at: https://pdxscholar.library.pdx.edu/open_access_etds

Part of the Speech and Hearing Science Commons Let us know how access to this document benefits you.

\section{Recommended Citation}

Ritchie, Pamela S., "Intelligibility of spondees via bone conduction at elevated presentation levels" (1990). Dissertations and Theses. Paper 4150.

https://doi.org/10.15760/etd.6034

This Thesis is brought to you for free and open access. It has been accepted for inclusion in Dissertations and Theses by an authorized administrator of PDXScholar. Please contact us if we can make this document more accessible: pdxscholar@pdx.edu. 
AN ABSTRACT OF THE THES IS OF Pamela S. Ritchie for the Master of Science in Speech Communication: Speech and Hearing Sciences presented October 29, 1990 .

Title: Intelligibility of Spondees via Bone Conduction at Elevated Presentation Levels.

APPROVED BY THE MEMBERS OF THE THESIS COMMITTEE:
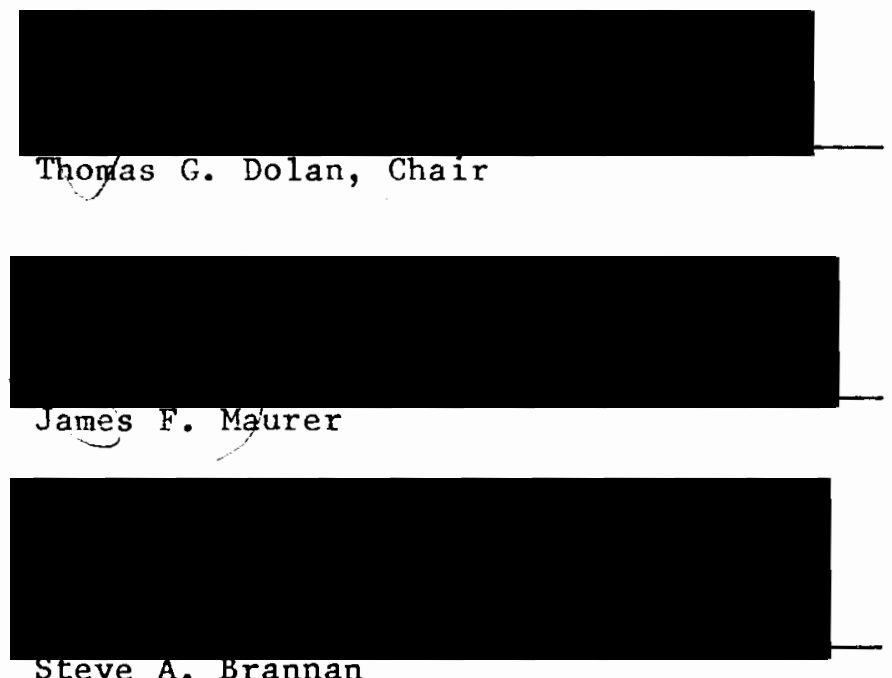

Speech reception threshold testing by bone conduction is very useful in diagnostic audiometry. However, there are little data regarding the CID $W-1$ spondee word 1 ists used with the Radioear $B-71$ and $\mathrm{B}-72$ and Pracitronic $\mathrm{KH} 70$ bone conduction vibrators at higher presentation levels for testing hearing impaired clients. Data are 
needed in order that results of speech reception thresholds using the CID W-1 word lists via these three bone conduction vibrators at higher intensity levels can be used confidently in the clinic. More data are available for speech reception thresholds using the CID $W-1$ word lists via earphones.

This study compared speech intelligibility presented via bone conduction at higher intensity levels to that presented via earphone at higher intensity levels.

Twelve normal hearing adults, with thresholds artificially elevated by binaural speech noise masking to simulate a hearing impairment, were used as subjects. Recorded CID W-1 spondee word 1 ists were presented via four transducers: Radioear B-71, Radioear B-72, and Pracitronic KH 70 bone vibrators, and a TDH-39 earphone.

Investigation of each bone conduction vibrator was accomplished by using forehead placement and using binaural speech noise masking via earphones. Investigation of the earphone was accomplished by simultaneously presenting binaural speech stimuli and speech noise masking.

Spondees from the CID $W-1$ word 1 ists were presented to the subject via a transducer. A bracketing technique was used to estimate the speech reception threshold. Using the intensity level of the predetermined SRT as $0 \mathrm{~dB}$, each 1 ist was presented at one of the following levels: $+4,+2,0,-2,-4$, and -6 .

Performance-intensity functions, the percentage of correctly identified spondees as a function of presentation level, were 
obtained for each transducer. The percentage of spondees correctly identified increased with the stimulus level for all transducers. Using linear regression, the line of best fit was calculated for each subject's data under each transducer condition. A one way analysis of variance indicated that there was a significant difference in the slopes of the performance-intensity functions of the transducers. A follow-up test for the one way analys is of variance indicated that there was a signficant difference between the TDH-39 earphone and each of the bone conduction vibrators. There were no significant differences among the bone conduction vibrators.

Results of the study suggested that CID $W-1$ word 1 ists and the 50 percent criterion may be inappropriate for use with these bone conduction vibrators at higher intensity levels. Given that there was a preponderance of data points below 50 percent for the bone vibrators, a criterion of less than 50 percent might be more appropriate. Because presentation levels might be elevated for sensorineural hearing impaired listeners, their SRT performances could be affected. Consequently, the results of speech reception threshold testing via bone conduction at higher intensity levels should be interpreted with caution in clinics. 
INTELLIGIBILITY OF SPONDEES VIA BONE CONDUCTION AT ELEVATED PRESENTATION LEVELS

by

PAMELA S. RITCHIE

A thesis submitted in partial fulfillment of the requirements for the degree of

MASTER OF SCIENCE

in

SPEECH COMMUNICATION:

SPEECH AND HEARING SCIENCES

Portland State University

1990 


\section{TO THE OFFICE OF GRADUATE STUDIES :}

The members of the Committee approve the thesis of Pamela $\mathrm{S}$. Ritchie presented October 29, 1990.
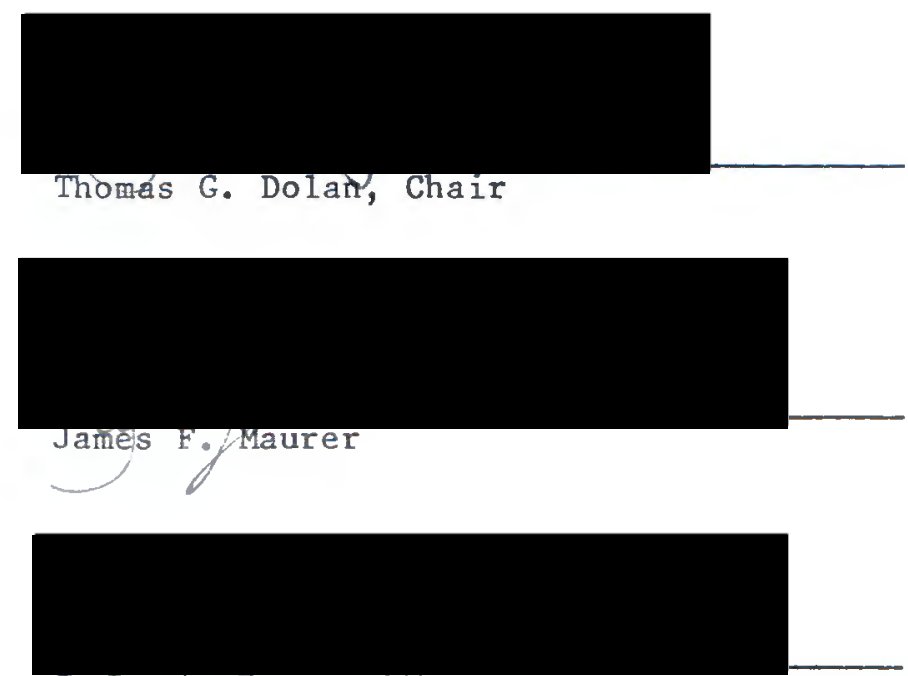

Steve A. Brannan

APPROVED:

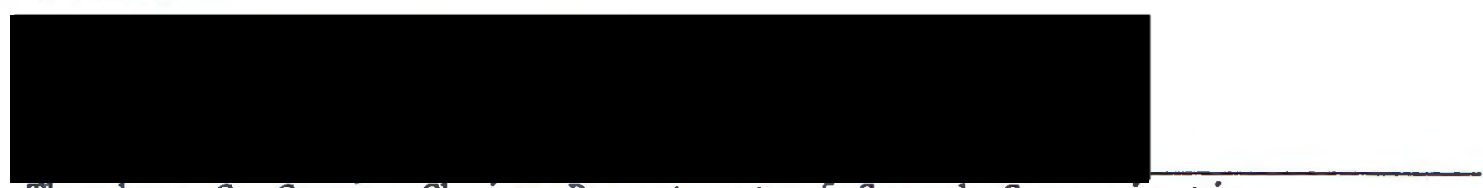

Theodore G. Grove, Chair, Department of Speech Communication

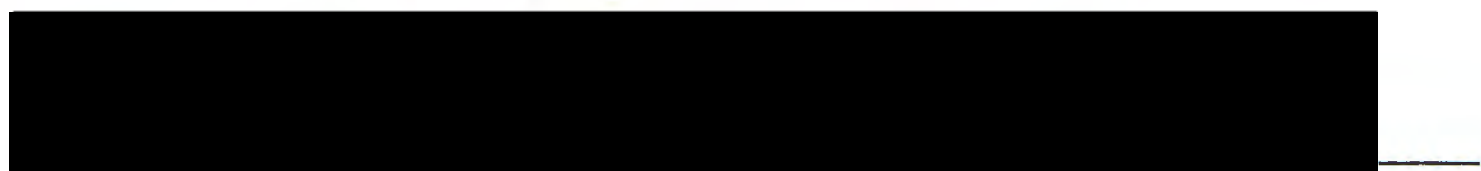

C. William Savery, Interim vice grovost for Graduate Studies and Research 


\section{ACKNOWLEDGEMENTS}

At this time, I extend my sincere thanks and gratitude to all my subjects, who remained my friends during and after the tedious and lengthy experimental testing sessions. I could not have completed my thesis without their help.

I thank Dr. Thomas Dolan for his continued help and guidance throughout every stage of this thesis. I also thank Dr. James Maurer and Dr. Steve Brannan for their constructive criticism during my thes is defense.

Thanks is extended to Eileen Mueller, secretary for the Speech and Hearing Sciences Department, who handled most of the peripheral details surrounding the experimental testing and the completion of my thesis.

Finally, I give my love and thanks to Lane Newman for his continued help and support throughout this endeavor and also throughout my years of graduate school. The many hours he uncomplainingly spent in involuntary solitude while I studied did not go unnoticed nor unappreciated. 
TABLE OF CONTENTS

PAGE

ACKNOWLEDGEMENTS . . . . . . . . . . . . . . . .

LIST OF TABLES . . . . . . . . . . . . . . . . . v v

LIST OF FIGURES ................... . . . vi

CHAPTER

I INTRODUCTION . . . . . . . . . . . . 1

II REVIEW OF THE LiteratURE . . . . . . . . . . 5

Speech Reception Threshold ......... 5

Speech via Bone Conduction ......... 7

III METHODS . . . . . . . . . . . . 13

Subjects ............... 13

Instrumentation and Stimuli ........ 13

Calibration ............. 14

Procedures .............. 15

IV RESULTS . . . . . . . . . . . . . 17

$\mathrm{V}$ DISCUSSION ..................... 23

REFERENCES . . . . . . . . . . . . . . . 26 


\section{LIST OF TABLES}

TABLE

PAGE

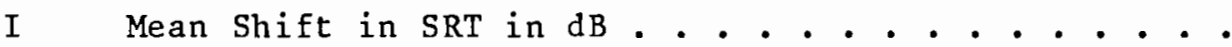

II Mean Slopes in Percentage of Spondees Correctly

Identified per $d B$ and Standard Deviations for Individual Data . . . . . . . . . . . .

III One Way Analysis of Variance for Slopes of Lines of Best fit . . . . . . . . . . . . . 21

IV Follow-Up Tests for the One Way Analysis of Variance

V Mean Pearson Product-Moment Correlation Coefficients

(Mean r) for Percentage of Spondees Correctly Identified Versus Intensity Level in $\mathrm{dB}$. . . 


\section{LIST OF FIGURES}

FIGURE

PAGE

1 Schematic Diagram of Instrumentation for (a)

Bone Conduction and (b) Air Conduction

Testing .....................

2 One Subject's Performance-Intensity Functions for

Four Transducers...............

3 Lines of Best Fit for Equalized Group Data for Four Transducers . . . . . . . . . . . . . 
CHAPTER I

INTRODUCTION

Speech reception threshold testing by bone conduction is very useful in diagnostic audiometry. Merre11, Wolfe, and McLemore (1973) found that bone conducted speech reception thresholds can be used to detect and determine the amount of conductive hearing losses in young children. Bone conducted speech reception thresholds can also be obtained for children when valid pure tone results cannot be measured. Their study also showed that bone conducted speech reception thresholds are in good agreement with pure tone air conduction thresholds. Valente and Stark (1977) found that bone conduction speech audiometry can aid in testing young children for whom pure tone results are unobtainable or unsatisfactory. Bone conducted speech reception thresholds can provide useful information when analyzing pure tone and speech air-bone gaps and when diagnosing a sensorineural hearing loss from a conductive one in young children. According to Goetzinger and Proud (1955), bone conduction speech audiometry has several advantages. Bone conduction speech testing results can verify the reliability of bone conducted pure tone results. Bone conduction speech testing is very useful in diagnosing conductive hearing losses in children, especially when valid pure 
tone thresholds are not obtainable. When speech reception testing is used in conjunction with speech discrimination testing, detailed information regarding sensorineural involvement can be obtained. According to Stockdell (1974), in adults there is a high correlation between bone conduction pure tone averages and speech reception thresholds. There is also a high correlation between air conduction pure tone averages and bone conduction speech reception thresholds. A high correlation also exists between air conduction speech reception thresholds and pure tone bone conduction thresholds. Information given by bone conduction speech reception thresholds and pure tone thresholds can help predict the success of otosclerosis surgery. Robinson and Kasden (1970) suggested using bone conduction speech discrimination scores to predict post-operative speech discrimination abilities for otosclerotic patients who have severe mixed hearing losses. Pre-operative air conduction speech discrimination scores are usually very low or nonexistent for these patients. However, pre-operative bone conduction speech discrimination scores are obtainable and correlate well to post-operative air conduction speech discrimination scores. The results indicated that preoperative bone conduction speech discrimination scores are good indicators of cochlear reserve and can be confidently used to predict post-operative air conduction speech discrimination for otosclerotic patients.

One operational definition of speech reception threshold is the dB leve 1 at which a 1 istener is able to correctly repeat 50 percent of the presented speech stimuli. Spondees are commonly used for 
speech stimuli. Hudgins, Hawkins, Karlin, and Stevens (1947) defined spondees as two-syllable words that are spoken with equal stress on each syllable. For research purposes, the speech reception threshold can be determined from a performance-intensity function. This is a graph of the percentage of correct responses (performance) on the vertical axis and the $d B$ intensity level on the horizontal axis. The slope of the curve is important in determining exactly where the 50 percent criterion leve 1 is reached (Egan, 1948). The steeper the slope and the narrower the intensity range in which the 1 istener responds, the more precisely the 50 percent criterion can be determined.

The spondees usually used for determining speech reception thresholds in adults are the Central Institute for the Deaf $\mathrm{W}-1$ spondee word lists, compiled by Hirsh, Davis, Silverman, Reynolds, Eldert, and Benson (1952). Even though the CID W-1 spondee word lists are commonly used for bone conduction speech reception thresholds, these lists were developed for speech via air conduction (Hirsh et al., 1952). Test performance, via bone conduction is presumed to be the same as that via air conduction at all intensity levels. Dolan and Morris (1989) demonstrated that the Radioear B-72 and Pracitronic KH 70 bone vibrators are comparable to the TDH-39 earphone in test performance at low intensity levels. This suggests that the CID W-1 spondee word lists are appropriate to use with these bone vibrators at low stimulus intensity levels. However, these two bone vibrators and the Radioear $B-71$ bone vibrator may be used clinically at higher intensity levels when testing hearing impaired 
clients. It is known that at high output levels, bone conduction vibrators demonstrate high distortion. If these bone vibrators are to be used for speech reception thresholds at higher intensity levels, their performance characteristics must be demonstrated to be similar to those for air conduction. This would then suggest that the CID W-1 spondee word lists are appropriate to use with the Radioear $B-71$ and $B-72$ and the Pracitronic $\mathrm{KH} 70$ bone conduction vibrators at higher stimulus intensity levels.

The purpose of this study is to determine if the spondee word lists are appropriate to use at high intensity levels with these three bone conduction vibrators. Performance-intensity functions for the Radioear $B-71$ and $B-72$ and Pracitronic $\mathrm{KH} 70$ bone vibrators will be determined at intensity levels above the normal speech reception thresholds. The slopes of these performance-intensity functions will then be compared to the slope of the performance-intensity function for air conduction at higher intensity levels using the TDH-39 earphone. Testing at higher intensity levels for the bone conduction vibrators will be accomplished by testing normal hearing adults using forehead placement and using speech noise masking to both ears via earphones. The masking noise will simulate a hearing loss in the subjects by raising their threshold values to higher intensity levels. This will allow data to be obtained for performanceintensity functions at higher intensity levels. 
CHAPTER II

REVIEW OF THE LITERATURE

SPEECH RECEPTION THRESHOLD

The term speech reception threshold was first used by Hughson and Thompson (1942) to describe the threshold of speech intelligibility because they felt that the latter term was too ambiguous. According to Hudgins et al. (1947), the speech stimuli used to determine speech reception threshold should possess the following characteristics:

(1) Familiarity: the words should be simple and familiar. The words should only test speech reception threshold and not vocabulary or intelligence.

(2) Phonetic dissimilarity: the words should not rhyme or sound similar because they would require even finer discrimination abilities from the listener.

(3) Normal sampling of English speech sounds: the words should be representative of speech shoulds used in English.

(4) Homogeneity with respect to basic audibility: all words should be understood equally as well when they are spoken at a constant level. Homogeneity is important so that the percentage of correct responses heard will rise sharply from zero to 100 percent in a narrow intensity range.

Egan (1948) stated that in order for test lists to detect small changes in speech intelligibility and be convenient, the test words 
should be equally difficult. If a test word is always missed or is always understood correctly by a listener, it should be omitted (Egan, 1948). If the test words are comparable in difficulty, then differences in listeners' scores will be due to the transducer that is used and not the word 1ist (Egan, 1948).

Hudgins et al. (1947) studied the steepness of performanceintensity functions for three different types of words: (a) spondees, (b) other two-syllable words, and (c) monosyllable words. Their findings demonstrated that the steepness of the function depended upon the type of words used. The steepest function was obtained with spondees. Therefore, the 50 percent criterion level of threshold was achieved at a lower $d B$ intensity level when using spondee word lists (Hudgins et al., 1947).

The spondee word 1 ists used in the above study were from a phonograph record of Harvard's Psycho-Acoustic Laboratory Auditory Test No. 9 (Hudgins et a1., 1947). Because of problems with word unfamiliarity and recording quality, modifications were made to the Auditory Test No. 9 by the Central Institute for the Deaf (Hirsh et a1., 1952). The new CID $W-1$ word 1 ists were recorded on magnetic tape, met a stricter criteria for word familiarity, and possessed a more limited vocabulary (Hirsh et al., 1952). Consequently, the improvements resulted in the performance-intensity function rising even more rapidly within an $8 \mathrm{~dB}$ intensity range. The 50 percent threshold is located at the steepest portion of the performanceintensity function (Hirsch et al., 1952). 
SPEECH VIA BONE CONDUCTION

Most of the literature regarding bone conduction testing has to do with calibration of instrumentation; a very small amount of the literature deals with speech reception threshold testing. Frank (1982) demonstrated that pure tone thresholds obtained with the Radioear B-7l bone conduction vibrator are higher at forehead placement than at mastoid placement. Beattie and Gager (1980) studied threshold force levels using spondee words for the Radioear B-70 and B-72 bone vibrators. The findings showed that the threshold force levels were comparable for the bone vibrators for a $1000 \mathrm{~Hz}$ calibration tone. However, the voltage levels needed to drive the bone vibrators to the threshold force level were not comparable. The study also investigated the output limits of the Radioear B-70 and B-72 bone conduction vibrators for bone conduction speech audiometry. The findings suggested that bone conduction speech reception thresholds can be obtained up to $70 \mathrm{~dB}$ HL before high amounts of distortion interfere with the results.

Frank and Crandell (1986) studied the amount of sound leakage (acoustic radiation) from the Radioear $B-71$ and $B-72$ and the Pracitronic KH 70 bone vibrators. Results indicated that the Pracitronic KH 70 bone vibrator had the smallest amount of acoustic radiation at $4000 \mathrm{~Hz}$, followed by the Radioear B-71 bone vibrator. The Radioear B-72 produced the largest amount of acoustic radiation. The Radioear $B-71$ and $B-72$ bone vibrators' amount of responsiveness at different frequencies was investigated by Richards and 
Frank (1982). Frequency response curves consisted of three resonant peaks which declined in intensity level as the frequency was increased. Because of output voltage variations at $4000 \mathrm{~Hz}$ by the B-72 bone vibrator, the findings questioned the currently accepted tolerance levels for calibration (Richards \& Frank, 1982).

Using an Oticon $A 20$ bone vibrator with a Bruel and $\mathrm{Kjaer}$ artificial mastoid, Srinivasan (1974) demonstrated a signficant correlation between bone conduction speech reception threshold and (a) air conduction speech reception threshold, (b) air conduction pure tone average, and (c) bone conduction pure tone average. In this study, Srinivasan (1974) also tested a normal hearing group, a conductive hearing loss group, and a sensorineural hearing loss group. A significant difference between the air conduction and bone conduction speech reception thresholds was found for the conductive hearing loss group. Air conduction and bone conduction speech reception thresholds were comparable for the normal hearing and sensorineural hearing loss groups. Occasionally, a noticeable difference between air and bone conduction speech reception thresholds was found for individuals with a noise-induced hearing loss. From these results, the author concluded that the oticon A 20 bone vibrator and the Bruel and $\mathrm{kjaer}$ artificial mastoid were useful clinically.

Edgerton, Danhauer, and Beattie (1977) investigated bone conduction speech reception thresholds and pure tone averages of the Radioear $\mathrm{B}-70 \mathrm{~A}$ bone vibrator. The results were compared to air conduction speech reception thresholds and pure tone averages and found to be in close agreement. The study also investigated the 
performance-intensity functions for spondee words presented via the Radioear $B-70 A$ bone vibrator. The findings demonstrated the Radioear B-70A bone vibrator's performance-intensity function was comparable to that via air conduction.

Valente and Stark (1977) studied bone conduction speech reception thresholds in young children using CID W-1 spondee word 1ists. The bone conduction speech reception threshold results showed that there is a $40 \mathrm{~dB}$ HL dial readout when routing the speech stimuli to the bone conduction vibrator. For a direct comparison of the bone conduction speech reception threshold to the pure tone average, a correction factor of $40 \mathrm{~dB}$ HL is subtracted from the bone conducted speech reception threshold. The study also found that bone conduction speech reception thresholds were very similar to bone conducted pure tone averages at $.5,1$, and $2 \mathrm{kHz}$ in children. Therefore, bone conducted pure tone averages can be predicted from bone conducted speech reception thresholds.

Stockde11 (1974) studied speech audiometry in adults. The findings showed a $38 \mathrm{~dB}$ HL dial readout when speech stimuli was routed through the bone conduction vibrator. Subtraction of a $38 \mathrm{~dB}$ correction factor from the bone conduction speech reception threshold was used for comparison to the pure tone average. Bone conduction pure tone averages and speech reception thresholds were highly correlated as were bone conduction pure tone averages and air conduction speech reception thresholds in adults. The high correlation suggests that the bone conduction pure tone averages may be a better indicator of speech reception thresholds for sensorineural hearing losses. Speech 
discrimination testing by bone conduction and air conduction was also investigated. However, because of presentation leve1, little correlation between the two was noted.

Barry and Gaddis (1978) investigated the output limitations of the Radioear $B-70 A$ bone conduction vibrator for adult speech discrimination scores. The mean percentage of correct responses began to decrease at $70 \mathrm{~dB} \mathrm{HL}$ and was 50 percent at $80 \mathrm{~dB}$ HL. At $90 \mathrm{~dB} \mathrm{HL}$ subjects became very uncomfortable from the heat and vibration emitted from the bone conduction vibrator. The study also investigated the transfer function for the Radioear B-70A bone conduction vibrator based on a $500 \mathrm{~Hz}$ calibration signal. The output became nonlinear after $50 \mathrm{~dB} \mathrm{HL}$. Significant total harmonic distortion was evident at $60 \mathrm{~dB} \mathrm{HL}$ and by $80 \mathrm{~dB} \mathrm{HL}$ was 100 percent. The study concluded that the maximum output for the Radioear B-70A bone conduction vibrator was $55 \mathrm{~dB}$ HL with less than 10 percent total harmonic distortion. Even though normal hearing subjects showed good speech discrimination up to $70 \mathrm{~dB} \mathrm{HL}$, individuals with sensorineural hearing losses or poor speech discrimination abilities would not be properly evaluated at levels above $55 \mathrm{~dB} \mathrm{HL}$.

Miller (1964) reported a technique for identifying feigned unilateral hearing losses by using bone conducted speech stimuli with air conduction masking noise. With forehead placement of the bone conduction vibrator, both cochlea may be stimulated. If air conduction masking noise is presented binaurally, the speech reception thresholds of both cochlea will be increased; if air conduction masking noise is presented monaurally, only the masked ear threshold 
will be increased. First a patient's bone conduction speech reception threshold level is determined. Binaural masking noise is presented and gradually increased during patient repetition of spondees. The leve 1 at which the patient stops responding is recorded. Monaural masking noise is next presented to the "poor" ear and gradua11y increased during patient repetition of spondees. The level at which the patient ceases responding is again recorded. Monaural masking noise is presented to the "better" ear and the same procedure is followed. Comparison of the results indicated the technique is good for determining true unilateral hearing losses and possibly aiding in determination of a conductive component to a hearing loss. Kasden and Robinson (1973) investigated bone conduction speech discrimination scores for patients with other conductive hearing loss pathologies. The authors wanted to determine if these scores would indicate the amount of cochlear reserve for this type of patient. The results showed little difference between air conduction and bone conduction scores for non-otosclerotic conductive hearing loss patients. The authors concluded that the difference between air and bone conduction scores for otosclerotic patients is due to ossicular fixation affecting the frequency response and impedance of the middle ear.

Robinson and Kasden (1977) studied otosclerotic patients who had undergone stapedectomies. Hearing evaluations performed one week after surgery indicated that these patients had mid to high frequency sensorineural losses and decreased speech discrimination scores when compared to pre-operative audiometric results. However, pre- and 
post-operative bone conduction speech discrimination scores remained very similar, indicating that cochlear reserve was maintained. The study indicated that comparison of pre- and post-operative bone conduction speech discrimination scores could reliably predict the outcome of stapedectomy surgery. 
CHAPTER III

METHODS

SUB JECTS

Twelve normal hearing adults were selected from the PSU student population. Their hearing thresholds, as determined by air and bone conduction testing, were $20 \mathrm{~dB} \mathrm{HL}$ or lower with no air-bone gaps greater than $10 \mathrm{~dB}$ (American National Standards Institute, 1969) at the frequencies .25, .5, 1, 2, 3, 4, 6, and $8 \mathrm{kHz}$.

\section{INSTRUMENTATION AND STIMULI}

Recorded CID W-1 word lists were used as stimuli. The six word lists were dubbed from $A$ to $F$ on cassette tapes from Auditec vinyl discs. The lists were comprised of the same 36 spondees that were arranged in different sequences. Instrumentation included the following in the order of connection: (a) a Proton 740 stereo cassette tape player for presentation of the CID W-1 word 1 ists, (b) a Beltone 2000 clinical audiometer, and (c) a transducer. The transducer was the TDH-39 earphone for obtaining air conduction speech reception thresholds and the Radioear B-71, Radioear B-72, or the Pracitronic KH 70 bone conduction vibrator for obtaining bone 
conduction speech reception thresholds. Figure 1 gives the schematic diagram of instrumentation for both bone and air conduction testing.

a.

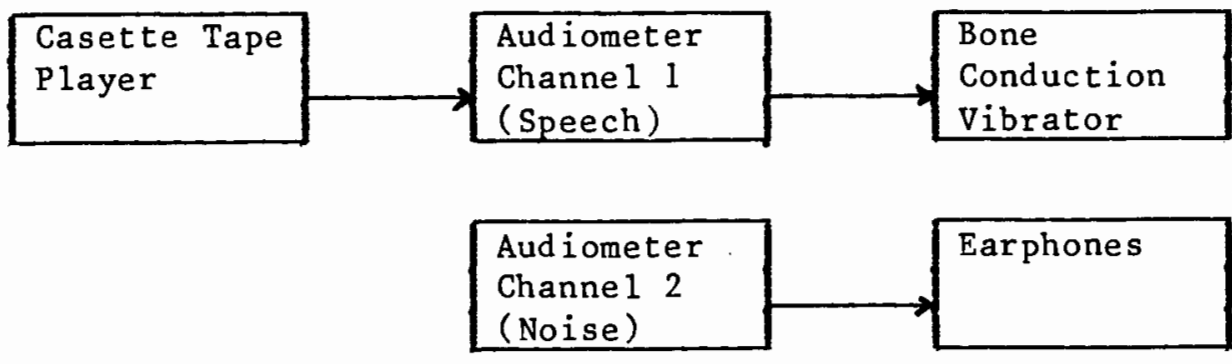

b.

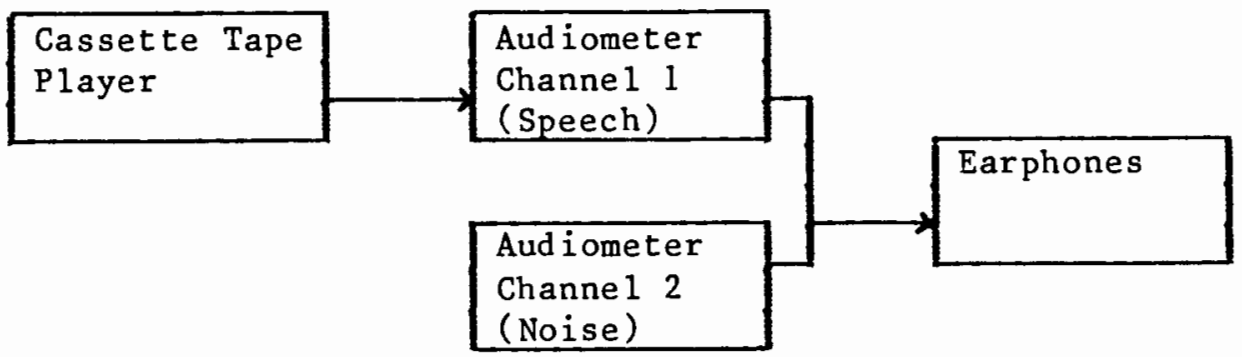

Figure 1. Schematic diagram of instrumentation for (a) bone conduction and (b) air conduction testing.

\section{CALIBRATION}

Pre- and post-session calibration of the TDH-39 earphone and the Radioear $\mathrm{B}-71$ and $\mathrm{B}-72$ and Pracitronic $\mathrm{KH} 70$ bone vibrators was performed with a Bruel and Kjaer sound level meter type 2230 and a Bruel and $\mathrm{Kjaer}$ artificial mastoid. A Bruel and $\mathrm{Kj}$ aer force gauge was used to monitor the amount of force coupling the bone vibrator to the forehead. The VU meter of the Beltone audiometer was checked each session with a $1000 \mathrm{~Hz}$ calibration tone recorded on a cassette tape to achieve the proper output levels. 


\section{PROCEDURES}

For the experimental testing, each subject was seated in a sound-treated room. Instructions were read to the subject. During bone conduction testing, TDH-39 earphones were placed on the subject's ears and a bone vibrator was placed on the subject's forehead. In order to raise the subject's speech reception threshold by bone conduction, binaural speech noise masking was used during presentation of speech stimuli. The bone vibrators were coupled to the forehead with a force of 550 grams (ANSI, 1969). During air conduction testing, TDH-39 earphones were placed on the subject's ears, and binaural speech noise masking was presented simultaneously with the binaural speech stimuli. The order of transducers was counterbalanced across subjects.

Spondees from the CID $\mathrm{W}-1$ word 1 ists were presented to the subject via a transducer. With the first randomly chosen word list, a bracketing technique was used to estimate the speech reception threshold (SRT). The bracketing technique consisted of presenting one spondee 8-10 dB above the subject's estimated SRT. If the subject responded correctly, a second spondee was presented at the same leve1. The subsequent presentations were attenuated in one dB steps with two spondees given at each level. When the subject missed one spondee, two more spondees were presented at the same leve1. The level where two out of four spondees were repeated correctly was then determined. This was considered the subject's estimated SRT. Then, using the intensity level of the pre-determined SRT as $0 \mathrm{~dB}$, each 
list was presented at one of the following levels: $+4,+2,0,-2$, -4 , and -6 . The order of levels was randomized for each subject. The number and percentage of correct responses were calculated for each subject.

Unmasked bone conduction SRTs were also obtained for each bone conduction vibrator with each subject. This information allowed calculation of the $d B$ amount that the threshold of speech intelligibility was raised for each bone conduction vibrator by the speech noise masking. 
CHAPTER IV

\section{RESULTS}

Masking noise was presented binaurally to 12 subjects at $40 \mathrm{~dB}$ HL in order to simulate a hearing loss and thereby raise the SRT. The amount of shift in SRT was determined for each listener by obtaining the estimated SRT in quiet and in noise and then subtracting the two estimates. The mean threshold shifts are given in Table I. The mean shift in SRT was greatest for the TDH-39 earphone $(36.5 \mathrm{~dB})$ and the mean shift in SRT was smallest for the Pracitronic KH 70 bone vibrator $(23 \mathrm{~dB})$.

TABLE I

MEAN SHIFT IN SRT IN dB

Mean Shift

Standard Deviation

$$
\frac{\text { Earphone }}{36.5}
$$

2.5

$$
\frac{\mathrm{KH} 70}{23.00}
$$$$
3.09
$$

$$
\frac{B-71}{24.50}
$$$$
4.49
$$

$2 \frac{B-72}{25.50}$

5.94

Note: The amount of shift in SRT was determined for each listener by obtaining the estimated SRT in quiet and in noise and then subtracting the two estimates $(n=12)$.

Figure 2 shows one subject's performance-intensity functions for the TDH-39 earphone, Radioear B-71 and B-72 bone conduction vibrators, and the Pracitronic $\mathrm{KH} 70$ bone conduction vibrator. The ordinate is the percentage of spondees correctly identified; the 
abscissa is the presentation level relative to the estimated SRT. The percentage of spondees correctly identified increased with the stimulus level for all transducers. The function for the earphone appears slightly steeper than those for the bone conduction vibrators. The poorest performance was seen for the B-72 bone vibrator, as demonstrated by a maximum of 50 percent at the highest presentation level.

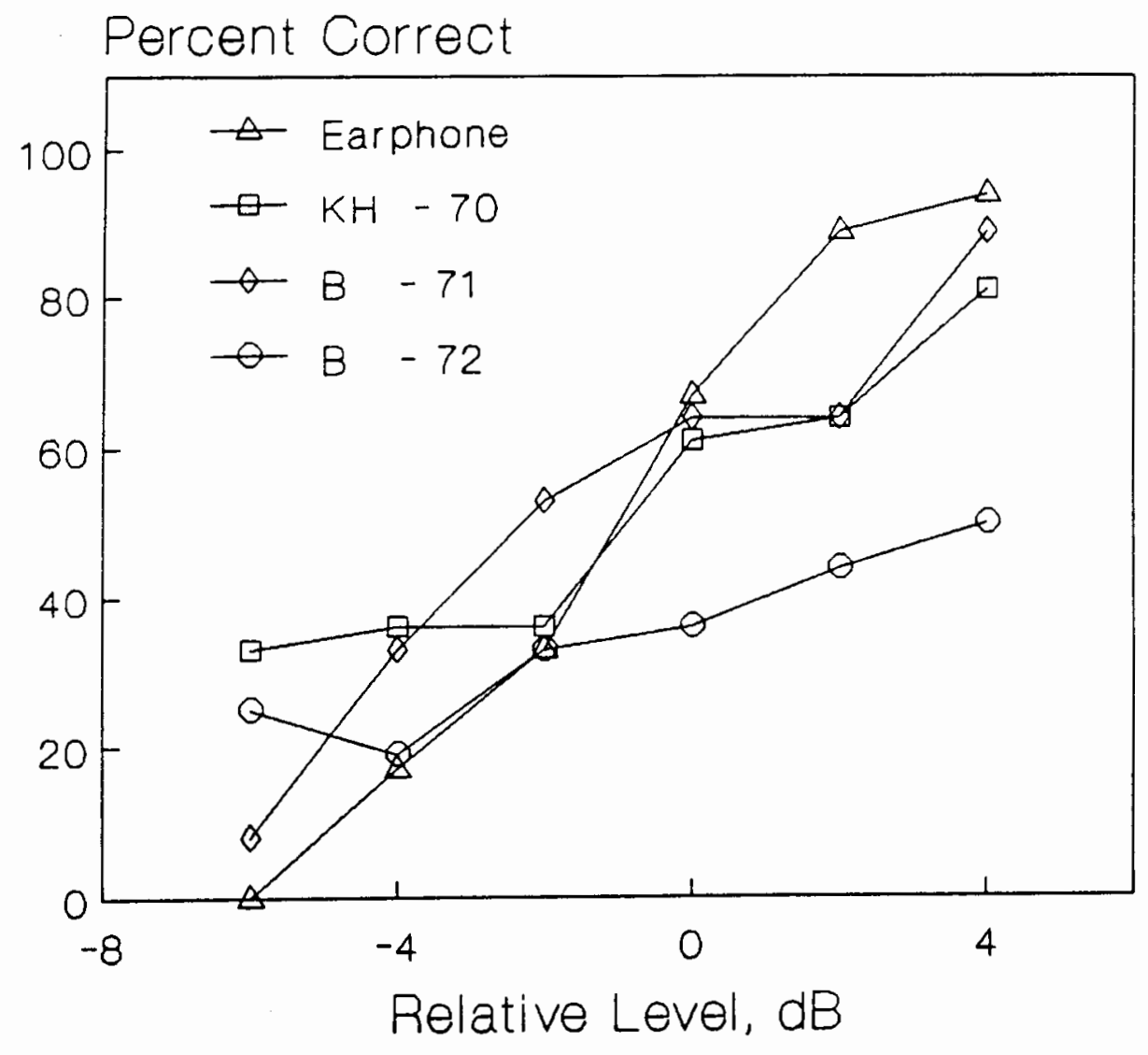

Figure 2. One subject's performance-intensity functions for four transducers. The ordinate is the percentage of spondees correctly identified and the abscissa is the presentation level, in $\mathrm{dB}$, relative to the estimated SRT. 
Using linear regression, the line of best fit was calculated for each subject's data under each transducer condition. The data were equalized along the $\mathrm{X}$ axis so that the 50 percent point of the line of best fit was aligned with $0 \mathrm{~dB}$. Figure 3 shows the resulting equalized group data for each transducer. The line of best fit to the group data is shown in each plot. Again, as the presentation level increased, the percentage of correct responses increased for each transducer.
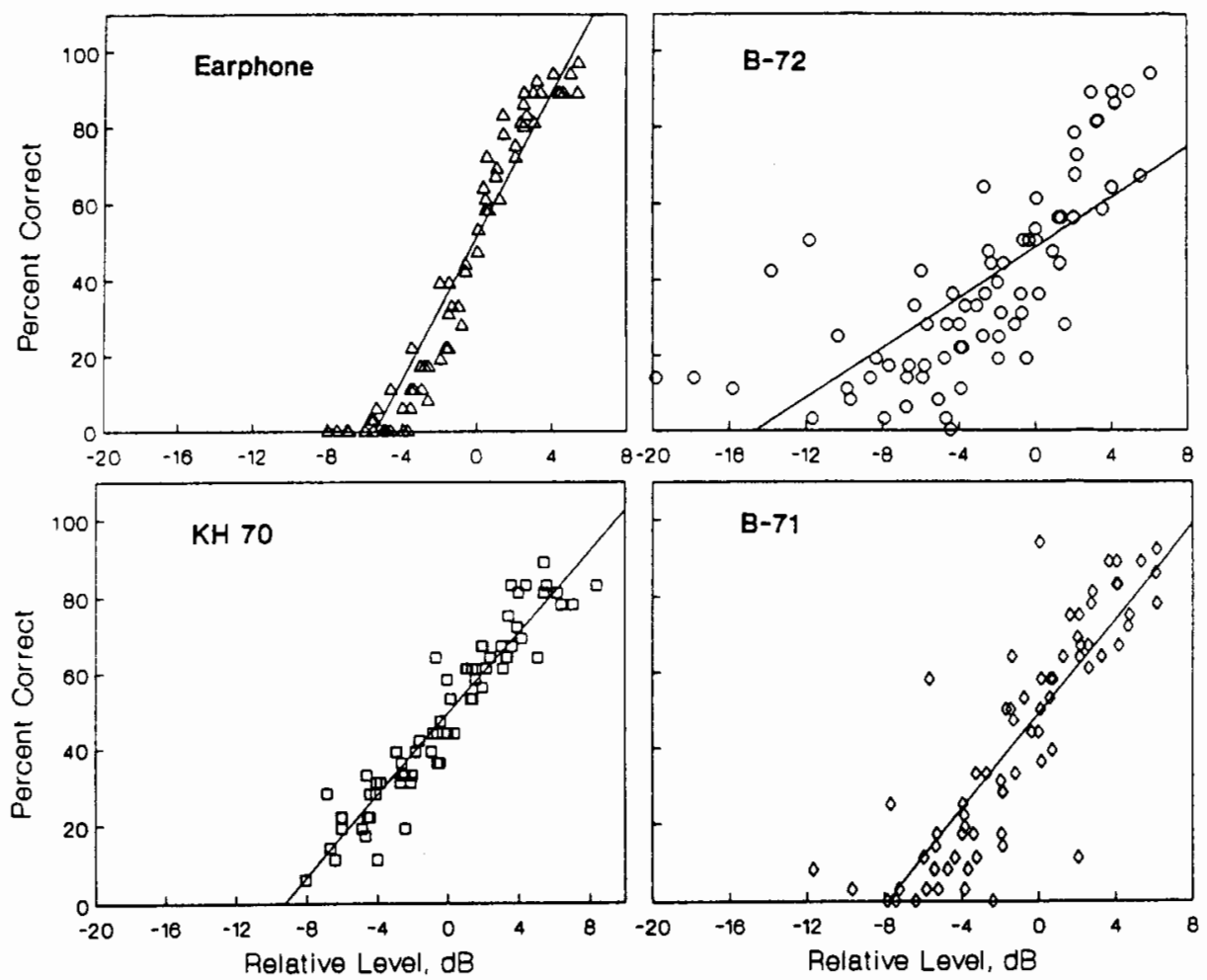

Figure 3. Lines of best fit for equalized group data for four transducers. The ordinate is the percentage of spondees correctly identified and the abscissa is the equalized (see text) presentation level, in $d B$. 
The mean slopes of lines of best fit to individual data are given in Table II. The highest slope is seen for the TDH-39 earphone (9.47 percent per $d B$ ). The lowest slope is seen for the Pracitronic KH 70 bone vibrator ( 5.49 percent per dB). Among the bone conduction vibrators, the slope is highest for the Radioear B-71 bone conduction vibrator $(7.26$ percent per $d B)$.

\section{TABLE II}

MEAN SLOPES IN PERCENTAGE OF SPONDEES CORRECTLY IDENTIFIED PER dB AND STANDARD DEVIATIONS FOR INDIVIDUAL DATA

\begin{tabular}{|c|c|c|c|c|}
\hline & Earphone & $\mathrm{KH} 70$ & $\mathrm{~B}-71$ & $\mathrm{~B}-72$ \\
\hline Mean Slope & 9.473 & 5.485 & 7.262 & 5.565 \\
\hline Standard Deviation & 1.025 & 1.006 & 1.535 & 2.251 \\
\hline
\end{tabular}

Note: $\mathbf{n}=12$

A one way analysis of variance indicated that there was a significant difference in the performance-intensity slopes of the transducers $(p<.05)$. A follow-up scheffé $F$ test indicated that there was a significant difference between the TDH-39 earphone and each of the bone conduction vibrators $(p<.05)$. There were no significant differences among the bone conduction vibrators. Tables III and IV show the results of the analysis.

The spread of data points clustered closely around the line of best fit for the $\mathrm{TDH}-39$ earphone and the Pracitronic $\mathrm{KH} 70$ bone vibrator. The greatest spread of data was seen for the Radioear B-72 bone vibrator. The mean Pearson product-moment correlation 
TABLE III

ONE-WAY ANALYSIS OF VARIANCE FOR SLOPES OF LINES OF BEST FIT One Factor ANOVA-Repeated Measures for $\mathrm{X}_{1} \cdot . \cdot \mathrm{X}_{4}$

Source $\quad \underline{\mathrm{df}}$ Sum of Squares Mean Square F-test $\underline{\text { P Value }}$

$\begin{array}{crrrrr}\text { Between Subjects } & 11 & 29.61 & 2.692 & .482 & .9021 \\ \text { Within Subjects } & 36 & 201.009 & 5.584 & & \\ \text { Treatments } & 3 & 126.294 & 42.098 & 18.594 & .0001 \\ \text { Residual } & 33 & 74.715 & 2.264 & & \\ \text { Total } & 47 & 230.619 & & & \end{array}$

Note: Reliability estimates for: all treatments $=-1.074$

single treatment $=-0.149$

TABLE IV

FOLIOW-UP TESTS FOR THE ONE-WAY ANALYSIS OF VARIANCE

One Factor ANOVA-Repeated Measures for $\mathrm{X}_{1} \cdot \mathrm{C} \cdot \mathrm{X}_{4}$

Comparison

Earphone vs. KH 70

Earphone vs. $B-71$

Earphone vs. $B-72$

KH 70 vs. $\mathrm{B}-71$

$\mathrm{KH} 70$ vs $\mathrm{B}-72$

$B-71$ vs $B-72$

\section{Mean}

Diff.

3.988

2.211

3.907

$-1.777$

$-0.081$

1.696
Fisher

PLSD

1. $25 *$

1. $25 *$

1. $25 *$

1. $25 *$

1.25

$1.25 \%$
Scheffé

F-Test

$14.048 *$

$4.318 *$

$13.485 *$

2.789

.006

2.542
Dunnett $t$

6.492

3.599

6.36

2.893

0.131

2.761

*Significant at 95 percent

coefficients, $r$, are given in Table $V$. The highest correlation was seen for the TDH-39 earphone (mean $r=.9682$ ), while the poorest correlation was seen for the Radioear $B-72$ bone vibrator (mean $\mathbf{r}=$ $.8833)$. 


\section{TABLE $V$}

MEAN PEARSON PRODUCT-MOMENT CORRELATION COEFFICIENTS (MEAN $r$ ) FOR PERCENTAGE OF SPONDEES CORRECTLY IDENTIFIED VERSUS INTENSITY LEVEL IN $d B$

Mean $r \quad \frac{\text { Earphone }}{.9682} \quad \frac{\mathrm{KH} 70}{.9506} \quad \frac{\mathrm{B}-71}{.9125} \quad \frac{\mathrm{B}-72}{.8833}$

Note: $\mathrm{n}=12$ 
CHAPTER V

DISCUSSION

This study compared the intelligibility of speech presented via bone conduction at higher intensity levels to that presented via earphone at higher intensity levels. Binaural speech noise masking was used to artifically elevate the speech reception thresholds of 12 subjects and simulate a hearing impairment.

Performance-intensity functions were obtained for Radioear B-71, Radioear B-72, Pracitronic KH 70 bone conduction vibrators, and a TDH-39 earphone. The performance-intensity function for each bone vibrator did not rise as steeply as the function for the earphone. The results indicated that there was a significant difference between the slope of the function for the TDH-39 earphone and for each of the bone conduction vibrators at higher intensity levels. of the three bone conduction vibrators, the performance-intensity function for the Radioear B-71 demonstrated the steepest slope. However, the results of the one way analysis of variance revealed no significant difference among the slopes for the three bone vibrators. According to Hirsh et al. (1952), the performance-intensity function, when using CID $W-1$ word lists, should rise at a rate of 8 
percent per $d B$ in an intensity range of $8 \mathrm{~dB}$. The steeper the slope and the narrower the intensity range between intelligibility and nonintelligibility of the word, the more accurately the SRT can be determined. The results of this study indicated that the slopes of the functions for the Radioear $B-71, B-72$, and Pracitronic $\mathrm{KH} 70$ bone conduction vibrators rose at a lower than desirable rate at higher intensity levels.

A possible explanation for the lower slopes could be distortion. Output distortion at higher intensity levels for bone conduction vibrators has been demonstrated by Bary and Gaddis (1978). A majority of subjects in the present study (62 percent) thought that speech was clearer and easier to understand through the earphone. The Radioear B-71 bone vibrator was judged to provide the clearest speech by 23 percent of the subjects, while each of the other bone vibrators was preferred by only 8 percent of the subjects.

The results of this study suggest that the CID $W-1$ word 1 ists and the 50 percent criterion might be inappropriate for use with these bone vibrators at higher intensity levels. Given that there was a preponderance of data points below 50 percent for the bone vibrators, a criterion of less than 50 percent might be more appropriate. Because presentation levels might be elevated for sensorineural hearing impaired listeners, their SRT performances could be affected. Consequently, the results of speech reception threshold testing via bone conduction at higher intensity levels should be interpreted with caution in clinics. 
These findings, performed at higher intensity levels, differ from those of Dolan and Morris (1989). Their study compared the intelligibility of speech presented via bone conduction to that presented via earphone at low intensity levels. The results of their study suggested that the CID W-1 word 1 ists are appropriate to use with the Radioear $B-72$ and the Pracitronic $\mathrm{KH} 70$ bone conduction vibrators at low intensity levels. Also, the mean slope for the earphone was slightly steeper than that in the findings of Dolan and Morris (1989). An explanation could be that the masking noise in the present study caused the subjects to focus on the task and thereby improve their performances.

To determine effects of distortion on speech intelligibility, this study should be repeated using other types of bone conduction vibrators, such as the oticon A 20 , so that their performanceintensity functions can be compared to that of the TDH-39 earphone. 


\section{REFERENCES}

American National Standards Institute. (1969). Specifications for audiometers (ANSI S3.6-1969). New York.

Barry, S., and Gaddis, S. (1978). Physical and physiological constraints on the use of bone-conduction speech audiometry. Journal of Speech and Hearing Disorders, 43, 220-226.

Beattie, R., and Gager, D. (1980). Bone-conduction speech testing: Calibration force and voltage levels for two vibrators. Ear and Hearing, 1 (2), 75-82.

Dolan, T., and Morris, S. (1989). Speech Delivered via Bone Conduction. Unpublished master's thesis, Portland State University, Portland, OR.

Edgerton, B., Danhauer, J., and Beattie, R. (1977). Bone conduction speech audiometry in normal subjects. Journal of the American Audiology Society, $\underline{3}, 84-87$.

Egan, J. (1948). Articulation testing methods. Laryngoscope, 58 (9), 955-991.

Frank, T. (1982). Forehead versus mastoid threshold differences with a circular tipped vibrator. Ear and Hearing, 3 (2), 91-92.

Frank, T., and Crande11, C. (1986). Acoustic radiation produced by $\mathrm{B}-71, \mathrm{~B}-72$, and $\mathrm{KH} 70$ bone vibrators. Ear and Hearing, $\underline{7}$ (5), $344-347$.

Goetzinger, C., and Proud, G. (1955). Speech audiometry by bone conduction. Archives of Otolaryngology, 62 (6), 632-635.

Hirsh, I., Davis, H., Silverman, S., Reynolds, E., Eldert, E., and Benson, R. (1952). Development of materials for speech audiometry. Journal of Speech and Hearing Disorders, 17 (2), $321-337$.

Hudgins, C., Hawkins, J., Karlin, J., and Stevens, S. (1947). The development of recorded auditory tests for measuring hearing loss for speech. Laryngoscope, 57, 57-89. 
Hughson, W., and Thompson, E. (1942). Correlation of hearing acuity for speech with discrete frequency audiograms. Archives of Otolaryngology, 36 (4), 526-540.

Kasden, S., and Robinson, M. (1973). Bone conduction speech discrimination in different pathologies. Journal of Auditory Research, $13,268-270$.

Merrell, H., Wolfe, D., and McLemore, D. (1973). Air and bone conducted speech reception thresholds. Laryngoscope, 83 (12), 1929-1939.

Miller, A. (1964). The use of masked bone-conducted speech as an aid in the detection of feigned unilateral hearing losses. Journal of Speech and Hearing Disorders, 29, 333-335.

Richards, W., and Frank, T. (1982). Frequency response and output variations of Radioear $B-71$ and $B-72$ bone vibrators. Ear and Hearing, $\underline{3}$ (1), 37-38.

Robinson, M., and Kasden, S. (1970). Bone conduction speech audiometry: A calibrated method to predict post-stapedectomy discrimination scores. Annals of Otolaryngology, Rhinology, and Laryngology, 79, 818-825.

Robinson, M., and Kasden, S. (1977). Bone conduction speech discrimination: An indication of cochlear function in the immediate postoperative period. Archives of Otolaryngology, 103, 238-240.

Srinivasan, K. (1974). Bone conducted speech reception threshold. Scand inavian Audiology, 3, 145-148.

Stockde11, K. (1974). Speech by bone conduction in diagnostic audiometry. Audecible, 23 (3), 100-109.

Valente, M., and Stark, E. (1977). Bone-conducted speech audiometry with normal-hearing and hearing-impaired children. Journal of Auditory Research, 17, 105-108. 